\title{
Parasitaemia and Its Relation to Hematological Parameters and Liver Function among Patients Malaria in Abs, Hajjah, Northwest Yemen
}

\author{
Mohamed Al-Salahy, ${ }^{1}$ Bushra Shnawa, ${ }^{2}$ Gamal Abed, ${ }^{1}$ Ahmed Mandour, ${ }^{3}$ and Ali Al-Ezzi ${ }^{4}$ \\ ${ }^{1}$ Zoology Department, Faculty of Science, Assiut University, Assiut 71516, Egypt \\ ${ }^{2}$ Biology Department, Faculty of Science, Soran University, Kurdistan, Iraq \\ ${ }^{3}$ Parasitology Department, Faculty of Medicine, Assiut University, Assiut 71516, Egypt \\ ${ }^{4}$ Biology Department, Faculty of Education, Aden University, Aden, Yemen \\ Correspondence should be addressed to Ali Al-Ezzi; dr.aliizy@gmail.com
}

Received 27 October 2015; Accepted 7 February 2016

Academic Editor: Lúcia Galvão

Copyright (C) 2016 Mohamed Al-Salahy et al. This is an open access article distributed under the Creative Commons Attribution License, which permits unrestricted use, distribution, and reproduction in any medium, provided the original work is properly cited.

\begin{abstract}
Plasmodium falciparum malaria is the most common infection in Yemen. The present study aims to investigate changes in hematological and hepatic function indices of P. falciparum infected individuals. This study included 67 suspected falciparum malarial patients attended in clinics and rural Abs Hospital (Tehama, Hajjah), Yemen, from October 2013 to April 2014. The diagnosis of malaria was confirmed by thick and thin film with Giemsa staining of malaria parasite. Hematological parameters and serum levels of aspartate transaminase (AST), alanine transaminase (ALT), alkaline phosphatase (ALP), and bilirubin (total and direct) as test indicators of liver function were studied. Patients with parasitaemia tended to have significantly lower hemoglobin, hematocrit, white blood cell count, lymphocytes, and platelets, compared with healthy normal subjects. Neutrophils levels were significantly higher in cases of falciparum malaria in comparison to healthy normal subjects. Serums AST, ALT, ALP, and bilirubin (total and direct) in falciparum malaria patients were significantly higher $(p<0.0001)$ than those of falciparum malaria of free individuals. Hematological and liver dysfunctions measured parameters were seen associated with moderate and severe parasitaemia infection. This study concludes that hematological and hepatic dysfunction parameters could be indicator of malaria in endemic regions.
\end{abstract}

\section{Introduction}

Malaria continues to be a great health problem in some of the most populated areas of the world. Infection is caused by a parasite of genus Plasmodium which is transmitted to human beings by infected female anopheles mosquito [1]. In Yemen the population at risk of malaria constitutes $81 \%$ of the total population, with estimated one million cases in 2009 [2]. P. falciparum is the predominant species in Yemen where it is responsible for more than $90 \%$ of the malaria cases, with only minimal cases caused by Plasmodium vivax [3]. Studies have revealed that hematologic and biochemical changes occur in malaria infected blood and there are common complications associated with this disease. Hematological changes that are associated with malaria infection include anemia, thrombocytopenia, and disseminated intravascular coagulation [46]. Changes in physicochemical parameters of $P$. falciparum infested blood may vary with level of malaria endemicity, presence of haemoglobinopathies, nutritional status, demographic factors, and level of malaria immunity $[7,8]$. The infection of liver cells by the sporozoites form of the malarial parasite can cause organ congestion, sinusoidal blockage, and cellular inflammation. These changes in hepatocytes can lead to the leakage of parenchymal (transaminases) and membranous (alkaline phosphatase) enzymes of the liver to the circulation. Hence increase in liver enzymes AST, ALT, and ALP observed in malaria infected patients also demonstrated that the serum activities of these liver enzymes increased with 
the increase in malarial parasite density. This change could confirm that the hepatic stage of the parasite's life cycle in human host is accompanied by significant perturbation in the hepatocyte's parenchyma and membrane leading to leakage of liver enzymes into the general circulation $[9,10]$.

Liver involvement in malaria is common in patients of severe malaria and may manifest as jaundice, hepatomegaly, and elevated liver enzymes like aspartate and alanine transaminases [11]. Hyperbilirubinemia, mainly unconjugated, is a common feature of falciparum malaria and is attributed to hemolysis of both parasitized and nonparasitized erythrocytes and partly due to liver damage [12]. Therefore, well-informed changes in blood parameters in malaria infection and hepatic function enable the clinician to establish reliable diagnosis and therapeutic interventions. Hence this research attempts to evaluate the changes in both hematological parameters and liver functions and in addition to evaluate the relationship between the levels of parasite density in blood and changes in hematological and hepatic function as biomarkers in falciparum malaria infected patients in Abs region of Yemen, which suffers from a highly malaria endemic.

\section{Materials and Methods}

2.1. Selection of Subjects. A total of 67 patients with malaria parasitaemia attending the clinics and Rural Hospital of Abs (Tehama, Hajjah), Yemen, were enrolled as the test subjects, who were reported ill with fever (temperature $>37.5^{\circ} \mathrm{C}$ ), headache, vomiting, chills, diarrhea, and other clinical signs. Patients of age 18-48 years were selected who confirmed to be infected with the falciparum malarial parasite by microscopic examination of Giemsa stained thin blood smears. Patient's selection was done by simple random sampling of males and females between October 2013 and April 2014. 30 normal subjects free from malaria were used as control. Patients with hepatitis, smokers, and those who are taking any antimalaria drugs were excluded from this study.

\subsection{Malarial Parasite Density Determination. P. falciparum} parasitaemia was determined in various blood smears stained by Giemsa stain. Parasitaemia was calculated based on WHO [13]: low (+) 1-10/100 fields, mild (++) 11-100/100 fields, moderate $(+++) 1-10 /$ one field, and high parasitaemia $(++++)$ $>10 /$ one field.

2.3. Sample Collection and Preparation. Sample collection and preparation: Venous blood was collected aseptically from the subjects using $5 \mathrm{~mL}$ disposable syringes. The blood samples were collected and $4 \mathrm{~mL}$ was transferred into plain bottles for the biochemical assays whereas the remaining $1 \mathrm{~mL}$ was transferred into EDTA bottles for malaria parasite tests. The blood samples in the plain bottles were allowed to clot and retract after which they were centrifuged at $3000 \mathrm{rpm}$ for $10 \mathrm{~min}$ and the serum was transferred into sterilized plain bottles for the biochemical analysis.
TABLE 1: Occurrence of $P$. falciparum malaria in parasitaemia patients by intensity of infections.

\begin{tabular}{lc}
\hline $\begin{array}{l}\text { Intensity of infection } \\
\text { (Parasitaemia) } N=67\end{array}$ & $\begin{array}{c}\text { Number of patients with } \\
\text { falciparum malaria }(\%)\end{array}$ \\
\hline Low $(+)$ & $11(16.4)$ \\
Mild $(++)$ & $14(20.9)$ \\
Moderate $(+++)$ & $23(34.3)$ \\
High $(++++)$ & $19(28.4)$ \\
\hline
\end{tabular}

2.4. Hematological Analysis. Whole blood samples were collected in EDTA tube for determination of hematological parameters including hemoglobin $(\mathrm{Hb})$ concentration, tWBC count, neutrophils, lymphocytes, platelets, and packed cell volume (PCV) using automated (SYSMX.KX-21n) hematology analyzer.

2.5. Assays of Liver Functions Parameters. Serum aspartate transaminase and alanine transaminase (ALT) parameters were determined using Roche/Hitachi cobas c systems automatically. This assay follows the recommendations of the IFCC but was optimized for performance and stability by $[14,15]$. Alkaline phosphatase (ALP) parameter was determined using Roche/Hitachi cobas c systems automatically by colorimetric assay in accordance with a standardized method. In the presence of magnesium and zinc ions, p-nitrophenyl phosphate is cleaved by phosphatases into phosphate and pnitrophenol [16].

Serum bilirubin concentration was determined using Roche/Hitachi cobas c systems automatically and total bilirubin was determined by Diazo method [17], while direct bilirubin was determined by Diazo method (special) [18].

2.6. Statistical Analysis. The data were expressed as mean \pm SE. The results were analyzed statistically using column statistics and one $t$-test. Correlation among the investigated parameters was tested by curves and regression using linear regression to test departure from linearity with runs test. These analyses were carried out using computer statistics Prism 3.0 Package (Graph and Software, Inc., San Diego, USA). The minimum level of statistical significance was set at $p<0.05,0.01$, or 0.001 .

\section{Result}

Of the 67 patients infected with P. falciparum 11 (16.4\%) had low intensity of infecting low $1+$ (1-10/100 fields), 14 (20.9\%) had mild intensity of infection $2+$ (11-100/100 fields), $23(34.3 \%)$ had moderate intensity of infection 3+ (1-10/one field), and 19 (28.4\%) had high intensity of infection 4+ (>10/one field) (Table 1). There were significant decreases in the mean values of the hemoglobin $(\mathrm{Hb})$, packed cell volume (PCV), total leucocyte counts (tWBC), lymphocytes, and platelets. Neutrophils were significantly higher in patients with falciparum malaria in comparison to healthy subjects (Table 2). Table 3 shows the relationship between parasite density and hemoglobin and platelets. Table 4 shows the 
TABLE 2: Comparison of hematological parameters between the cases of $P$. falciparum malaria infection and healthy subjects.

\begin{tabular}{lcc}
\hline \multirow{2}{*}{ Parameters } & Patients with malaria & Healthy control subjects \\
& $N=67$ & $N=30$ \\
\hline $\mathrm{Hb}(\mathrm{g} / \mathrm{dL})$ & $9.4 \pm 0.28^{* * *}$ & $13.08 \pm 0.36$ \\
$\mathrm{PCV}(\%)$ & $29.47 \pm 0.82^{* * *}$ & $42.52 \pm 0.86$ \\
$\mathrm{~T} . \mathrm{WBC}\left(10^{3} / \mathrm{uL}\right)$ & $5.91 \pm 0.35^{* *}$ & $7.95 \pm 0.60$ \\
Lymphocytes $(\%)$ & $24.64 \pm 1.63^{* * *}$ & $39.17 \pm 2.19$ \\
Neutrophils $(\%)$ & $71.28 \pm 1.60^{* * *}$ & $48.46 \pm 2.30$ \\
Platelet $\left(\times 10^{3} / \mu \mathrm{L}\right)$ & $116.6 \pm 7.03^{* * *}$ & $353.4 \pm 18.72$ \\
\hline
\end{tabular}

${ }^{* *} p<0.01 ;{ }^{* * *} p<0.0001$.

TABLE 3: The level of hemoglobin and platelets in patient $P$. falciparum malaria with parasitaemia.

\begin{tabular}{lcc}
\hline Parasitaemia/field & Mean hemoglobin & Mean platelets \\
\hline Low $(+)$ & $12.75 \pm 0.2$ & $177.5 \pm 5.1^{* * *}$ \\
Mild $(++)$ & $10.95 \pm 0.1^{* * *}$ & $131.5 \pm 7.8^{* * *}$ \\
Moderate $(+++)$ & $8.64 \pm 0.3^{* * *}$ & $83.8 \pm 2.6^{* * *}$ \\
High $(++++)$ & $8.14 \pm 0.4^{* * *}$ & $53.1 \pm 6.9^{* * *}$ \\
Healthy & $13.08 \pm 0.3$ & $353.4 \pm 18.7$ \\
\hline
\end{tabular}

$* * *=p<0.0001$.

TABLE 4: Changes in liver functions biomarkers of the patient's $P$. falciparum malaria and healthy subjects.

\begin{tabular}{lcc}
\hline Parameters & Patients with malaria & Healthy subjects \\
& $N=67$ & $N=30$ \\
\hline $\begin{array}{l}\text { Aspartate aminotransferase } \\
\text { (AST) U/L }\end{array}$ & $51.12 \pm 2.85^{* * *}$ & $29.20 \pm 1.50$ \\
$\begin{array}{l}\text { Alanine aminotransferase } \\
\text { (ALT) U/L }\end{array}$ & $37.92 \pm 2.04^{* * *}$ & $20.97 \pm 1.42$ \\
$\begin{array}{l}\text { Alkaline phosphatase } \\
\text { (ALP) U/L }\end{array}$ & $177.4 \pm 10.49^{* *}$ & $108.2 \pm 6.81$ \\
$\begin{array}{l}\text { Total bilirubin (TB) umol/L } \\
\text { Direct bilirubin (DB) } \\
\text { umol/L }\end{array}$ & $70.13 \pm 7.04^{* * *}$ & $6.15 \pm 0.39$ \\
\hline
\end{tabular}

${ }^{* *} p<0.01{ }^{* * *} p<0.0001$.

changes in liver function biomarkers of the patient's $P$. falciparum malaria and healthy subjects. There were significant increases in the mean activity enzymes values of the AST, ALT, ALP, and serum total and direct bilirubin. Table 5 shows the relationship between parasite density and liver enzymes activities in serum. Level of parasitaemia correlates positively with mean liver enzyme activities specifically moderate and high parasitaemia showing higher values of liver enzyme activities when compared with patients having low and mild parasitaemia. Total bilirubin and direct bilirubin showed increase with increase in severity of parasitaemia of malarial infections (Table 6).

\section{Discussion}

In this study, hemoglobin $(\mathrm{Hb})$ and packed cell volume (PCV) decreased in P. falciparum affected patients compared to the healthy subjects. This finding agrees with previous reports $[5,19]$. The earlier studies reported a significant reduction in hemoglobin concentration and packed cell volume in patients with malaria parasitaemia. In this study the mean hemoglobin concentration showed $9.3 \mathrm{~g} / \mathrm{dL}$, but, in the study done by Nadeem et al. [20], hemoglobin level in $P$. falciparum affected patients was $13.7 \mathrm{gm} / \mathrm{dL}$. This value was more than that observed in our study. Reduced hemoglobin in malaria may be attributed to the increase of breakdown red blood cells by the parasites [21]. According to the report of Maina et al., [5] as contained in the National Guidelines for Diagnosis Treatment and Prevention of Malaria for Health Workers in Kenya, anemia is defined as $[\mathrm{Hb}]<10 \mathrm{~g} / \mathrm{dL}$ for both males and females. Furthermore, severe malarial anemia is defined as $[\mathrm{Hb}]<5.5 \mathrm{~g} / \mathrm{dL}$. Therefore, the drop in hemoglobin concentrations in the malarious subjects in our study ranged between 5 and $10.5 \mathrm{~g} / \mathrm{dL}$, ranging approximately from mild to moderate anemia. In study done by Bakhubaira [22], it was observed that anemia is more common amongst the patients infected by P. falciparum in Aden, Yemen.

Lower mean value for leukocyte count in the falciparum malaria patients compared to the healthy subjects was observed in our study. This was in concordance with other studies [23]. Similarly, decrease in WBC in malaria patients has been observed by S. Chandra and H. Chandra [24], who reported that low leukocyte count may be used as probable indicator for malaria in endemic countries. The present study reports a significant reduction in lymphocytes level in individuals infected with $P$. falciparum as compared to those not infected (healthy subjects). Reduction in the counts of lymphocytes was observed in some studies [25]. The decrease in lymphocyte counts associated with malaria may be due to reflecting redistribution of lymphocytes with sequestration in the spleen [26].

The present study reports a significant increase in neutrophil level of individuals infected with P. falciparum as compared to those not infected. These findings are in agreement with previous reports $[5,25]$. Increase of neutrophil in these cases could be a representation of early release of neutrophil from the bone in response to the infection. In our study, thrombocytopenia emerged as a predictor of malaria which is an observation of many studies which confirm it $[19,27,28]$. Low platelet count is a finding of malarial infection and thrombocytopenia may be more common than anemia in malaria infection.

The results reported in this study show some significant increases in activities enzymes aspartate transaminase (AST), alanine transaminase (ALT), and alkaline phosphatase (ALP) among patients with $P$. falciparum malaria. The increased serum levels of hepatic enzymes, transaminases (AST and ALT), and ALP are the biomarkers of liver disorders. Our results are consistent with other studies which reported that majority of the patients show elevation in serum activities (AST, ALT, and ALP) indicating liver damage [29, 30]. The increases in serum level of hepatic enzymes, transaminases (SGOT and SGPT), and alkaline phosphatase are the markers of liver damage. Ignatius et al. [31] reported that the liver enzymes leakage and bilirubin increased with increase in malaria parasite density. Usually, in uncomplicated malaria, 
TABLE 5: Mean liver enzymes levels in parasitaemia P. falciparum malaria and healthy control subjects.

\begin{tabular}{lccc}
\hline Mean parasitaemia/field & AST level (U/L) & ALT level (U/L) & ALP level (U/L) \\
\hline Low (+) & $28.36 \pm 1.04$ & $22.52 \pm 1.52$ & $95.69 \pm 4.88$ \\
Mild (+) & $34.26 \pm 1.45^{*}$ & $25.89 \pm 2.34$ & $137.6 \pm 9.73^{*}$ \\
Moderate (+) & $58.63 \pm 3.17^{* * *}$ & $47.27 \pm 1.73^{* * *}$ & $194.0 \pm 15.06^{* * *}$ \\
High (+) & $96.03 \pm 6.21^{* * *}$ & $60.28 \pm 5.58^{* * *}$ & $228.5 \pm 23.97^{* * *}$ \\
Healthy subjects & $29.24 \pm 1.04$ & $20.90 \pm 0.98$ & $108.2 \pm 4.80$ \\
\hline
\end{tabular}

${ }^{*} p<0.01 ;{ }^{* * *} p<0.000$.

TABle 6: Mean total and direct bilirubin levels in parasitaemia $P$. falciparum malaria and healthy control subjects.

\begin{tabular}{lcc}
\hline Parasitaemia & TB level (umol/L) & DB level (umol/L) \\
\hline Low $(+)$ & $8.54 \pm 0.68^{* *}$ & $2.48 \pm 0.16$ \\
Mild $(+)$ & $19.24 \pm 1.46^{* * *}$ & $5.27 \pm 0.35^{* * *}$ \\
Moderate $(+)$ & $68.31 \pm 6.45^{* * *}$ & $23.53 \pm 4.13^{* * *}$ \\
High $(+)$ & $137.6 \pm 13.25^{* * *}$ & $31.82 \pm 3.88^{* * *}$ \\
Healthy subjects & $6.12 \pm 0.27$ & $3.33 \pm 0.26$ \\
\hline$* * 0.001 ;{ }^{* * *} p<0.0001$ &
\end{tabular}

raised bilirubin is mainly due to hemolysis of parasitized and nonparasitized RBC and/or hepatocytes damage [32]. In this study raised bilirubin was mainly due to hepatic dysfunction. Molyneux et al. [33] suggested that jaundice, which may be deep, is usually accompanied by only moderate elevation of hepatic enzymes and results more from hemolysis than from hepatic damage. In the present study, the cause of jaundice was attributed mainly to intravascular hemolysis and hepatic dysfunction (42.1\%), as reported by other studies [34, 35].

\section{Conclusion}

Hemoglobin, platelets count, lymphopenia, tWBC, and neutrophilia were seen associated with mild, moderate, and severe parasitaemia infection. Acute falciparum malaria infection is associated with an increase in serum activity of aspartate and alanine aminotransferases and alkaline phosphatase thus indicating that the infection is associated with acute liver injury.

\section{Ethical Approval}

Ethical approval was given by the Hospital Management and Center of Malaria in Abs area.

\section{Conflict of Interests}

The authors declare that there is no conflict of interests regarding the publication of this paper.

\section{Acknowledgments}

The authors thank the Department of Zoology, Faculty of Sciences in Assiut University, for their cooperation and Aden
University for financial support for the study during the research period.

\section{References}

[1] K. Park, Park's Textbook of Preventive and Social Medicine, Banarsidas Bhanot, Jabalpur, India, 21st edition, 2011.

[2] WHO, Guidelines for the Treatment of Malaria, World Health Organization, Geneva, Switzerland, 2nd edition, 2010.

[3] M. Q. A. M. Abdulsalam, A. K. M. Mohammed, A. A. Ahmed, and M. Y. Fong, "Clinical situation of endemic malaria in Yemen," Tropical Biomedicine, vol. 27, no. 3, pp. 551-558, 2010.

[4] C. A. Facer, "Hematological aspects of malaria," in Infection and Hematology, Butterworth Heinemann, Oxford, UK, 1994.

[5] R. N. Maina, D. Walsh, C. Gaddy et al., "Impact of Plasmodium falciparum infection on haematological parameters in children living in Western Kenya," Malaria Journal, vol. 9, no. 3, article S4, 2010.

[6] S. Chandra and H. Chandra, "Role of haematological parameters as an indicator of acute malarial infection in Uttarakhand state of India," Mediterranean Journal of Hematology and Infectious Diseases, vol. 5, no. 1, Article ID e2013009, 2013.

[7] R. N. Price, J. A. Simpson, F. Nosten et al., "Factors contributing to anemia after uncomplicated falciparum malaria," The American Journal of Tropical Medicine and Hygiene, vol. 65, no. 5, pp. 614-622, 2001.

[8] L. M. Erhart, K. Yingyuen, N. Chuanak et al., "Hematologic and clinical indices of malaria in a semi-immune population of Western Thailand," American Journal of Tropical Medicine and Hygiene, vol. 70, no. 1, pp. 8-14, 2004.

[9] C. Burtis, E. Ashwood, and B. Border, "Liver functions," in Tietz Fundamentals of Clinical Chemistry, pp. 748-770, Saunders Company, Philadelphia, Pa, USA, 5th edition, 2001.

[10] I. Onyesom, "Activities of some liver enzymes in serum of $P$. falciparum malarial infected humans receiving artemisinin and non-artemisinin-based combination therapy," Annals of Biological Research, vol. 3, no. 7, pp. 3097-3100, 2012.

[11] R. Baheti, P. Laddha, and R. S. Gehlot, "Liver Involvement in falciparum malaria-a histo-pathological analysis," Journal, Indian Academy of Clinical Medicine, vol. 4, no. 1, pp. 34-38, 2003.

[12] T. Harinasuta and D. Bunnag, "The clinical features of malaria," in Malaria: Principles and Practice of Malariology, W. $\mathrm{H}$. Wernsdorfer and I. A. McGregor, Eds., vol. 1, pp. 709-732, Churchill Livingstone, Edinburgh, UK, 1988.

[13] WHO, Basic Malaria Microscopy: Part I Learner's Guide, World Health Organization, Geneva, Switzerland, 1991.

[14] H. U. Bergmeyer, M. Horder, and R. Rej, "Approved recommendation on IFCC methods for the measurement of catalytic 
concentration of enzymes. Part 2. IFCC method for aspartate aminotransferase," Journal of Clinical Chemistry and Clinical Biochemistry, vol. 24, pp. 497-510, 1986.

[15] ECCLS, "Determination of the catalytic activity concentration in serum of L-aspartate aminotransferase (EC 2.6.1.1, ASAT)," Klinische Chemie Mitteilungen, vol. 20, pp. 198-204, 1989.

[16] N. W. Tietz, A. D. Rinker, and L. M. Shaw, "IFCC methods for the measurement of catalytic concentration of enzymes .5. Ifcc method for alkaline-phosphatase," Journal of Clinical Chemistry and Clinical Biochemistry, vol. 21, no. 11, pp. 731-748, 1983.

[17] H. T. Malloy and K. A. Evelyn, "The determination of bilirubin with the photoelectric colorimeter," The Journal of Biological Chemistry, vol. 119, pp. 481-490, 1937.

[18] A. W. Wahlefeld, G. Herz, and E. Bernt, "Modification of Malloy-Evelyn method for a simple, reliable determination of total bilirubin in serum," Scandinavian Journal of Clinical and Laboratory Investigation, vol. 29, supplement 126, pp. 11-12, 1972.

[19] O. I. George and C. S. Ewelike-Ezeani, "Haematological changes in children with malaria infection in Nigeria," Journal of Medicine and Medical Sciences, vol. 2, no. 4, pp. 768-771, 2011.

[20] M. Nadeem, N. Ali, and M. A. Qamar, "Hematological findings in acute malarial infection list of authors along with highest qualification and institute," Biomedica, vol. 18, pp. 62-65, 2002.

[21] O. N. Goselle, C. O. E. Onwuliri, and V. A. Onwuliri, "Malaria infection in HIV/AIDS patients and its correlation with packed cell volume (PCV)," Journal of Vector Borne Diseases, vol. 46, no. 3, pp. 205-211, 2009.

[22] S. Bakhubaira, "Hematological parameters in severe complicated Plasmodium falciparum malaria among adults in Aden," Turkish Journal of Hematology, vol. 30, no. 4, pp. 394-399, 2013.

[23] I. S. Koltas, H. Demirhindi, S. Hazar, and K. Ozcan, "Supportive presumptive diagnosis of Plasmodium vivax malaria: thrombocytopenia and red cell distribution width," Saudi Medical Journal, vol. 28, no. 4, pp. 535-539, 2007.

[24] S. Chandra and H. Chandra, "Role of haematological parameters as an indicator of acute malarial infection in Uttarakhand state of India," Mediterranean Journal of Hematology and Infectious Diseases, vol. 5, no. 1, Article ID e2013009, 2013.

[25] O. T. Kayode, A. A. A. Kayode, and O. O. Awonuga, "Status of selected hematological and biochemical parameters in malaria and malaria-typhoid co-infection," Journal of Biological Sciences, vol. 11, no. 5, pp. 367-373, 2011.

[26] S. N. Wickramasinghe and S. H. Abdulla, "Blood and bone marrow changes in malaria," Best Practice \& Research Clinical Haematology, vol. 13, no. 2, pp. 277-299, 2000.

[27] T. B. Lathia and R. Joshi, "Can hematological parameters discriminate malaria from nonmalarious acute febrile illness in the tropics?" Indian Journal of Medical Sciences, vol. 58, no. 6, pp. 239-244, 2004.

[28] A. M. Malik, N. Zaffar, N. Ali, A. M. Malik, and R. Khan, "Haematological findings and endemicity of malaria in Gadap region," Journal of the College of Physicians and Surgeons Pakistan, vol. 20, no. 2, pp. 112-116, 2010.

[29] I. Oyewole, S. Senusie, and M. Mansaray, "Plasmodium falciparum-induced kidney and liver dysfunction in malaria patients in Freetown, Sierra Leone," Sierra Leone Journal of Biomedical Research, vol. 2, no. 1, pp. 70-74, 2010.

[30] I. Onyesom and N. Onyemakonor, "Levels of parasitaemia and changes in some liver enzymes among malarial infected patients in Edo-Delta Region of Nigeria," Current Research Journal of Biological Sciences, vol. 3, no. 2, pp. 78-81, 2011.
[31] C. M. Ignatius, E. N. Emeka, and N. E. Blessing, "Effect of malaria parasitaemia on liver enzyme tests," International Journal of Tropical Medicine, vol. 3, no. 3, pp. 49-52, 2008.

[32] A. H. Abro, A. M. Ustadi, H. A. Abro, A. S. Abdou, N. J. Younis, and S. I. Akaila, "Jaundice with hepatic dysfunction in P. falciparum malaria," Journal of the College of Physicians and Surgeons Pakistan, vol. 19, no. 6, pp. 363-366, 2009.

[33] M. E. Molyneux, S. Looareesuwan, I. S. Menzies et al., "Reduced hepatic blood flow and intestinal malabsorption in severe falciparum malaria," The American Journal of Tropical Medicine and Hygiene, vol. 40, no. 5, pp. 470-476, 1989.

[34] A. Bhalla, V. Suri, and V. Singh, "Malarial hepatopathy," Journal of Postgraduate Medicine, vol. 52, no. 4, pp. 315-320, 2006.

[35] R. Singh, M. Kaur, and D. A. Arora, "Prospective study of hepatic involvement in Plasmodium falciparum malaria," Journal of Clinical and Diagnostic Research, vol. 4, no. 2, pp. 2190-2197, 2010. 


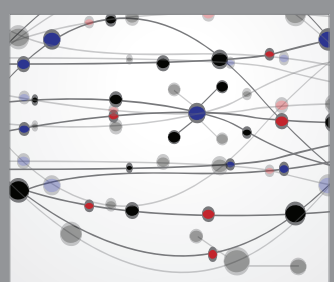

The Scientific World Journal
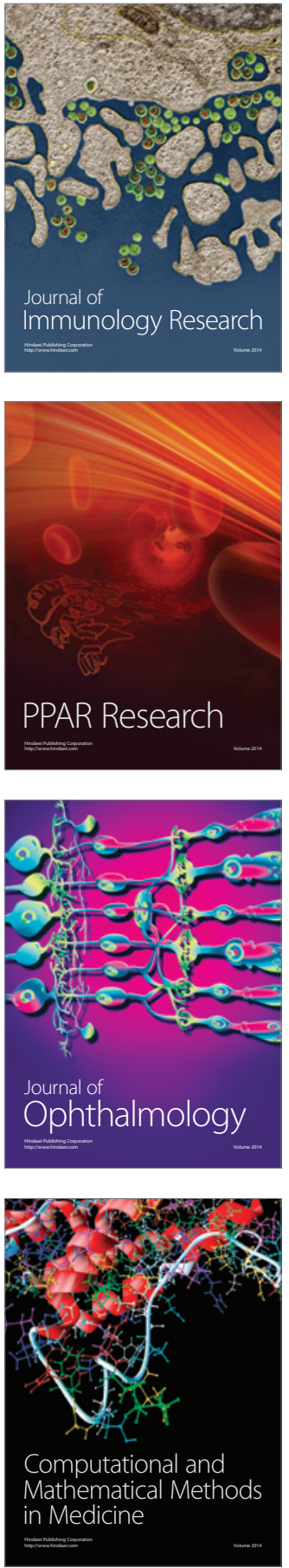

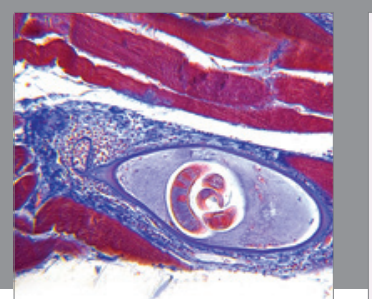

Gastroenterology Research and Practice

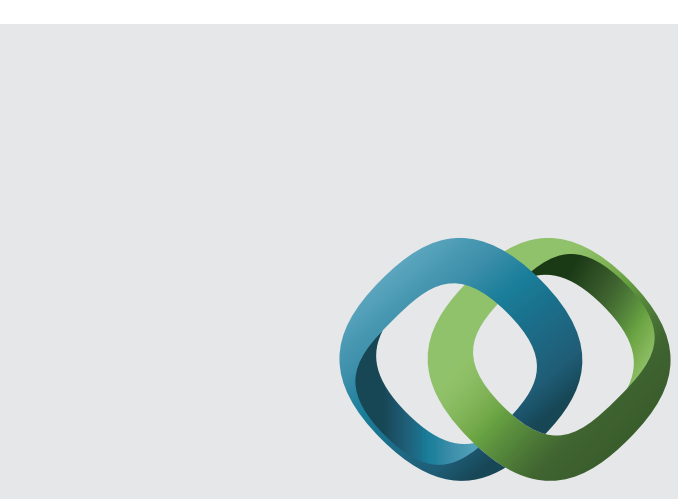

\section{Hindawi}

Submit your manuscripts at

http://www.hindawi.com
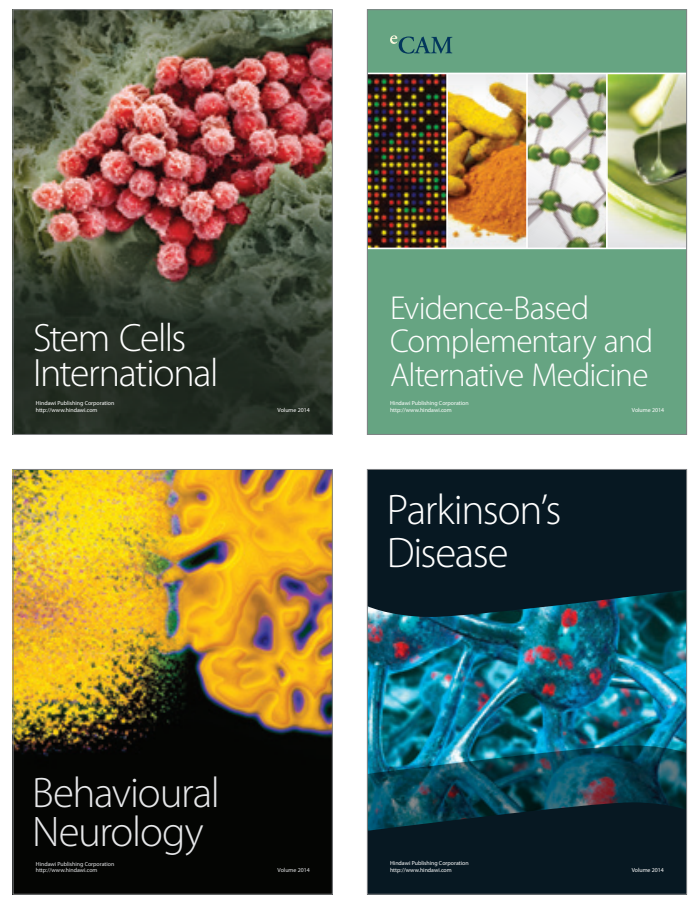
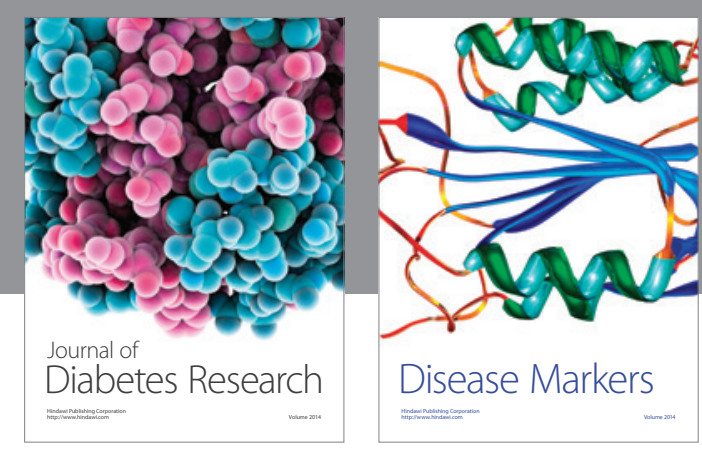

Disease Markers
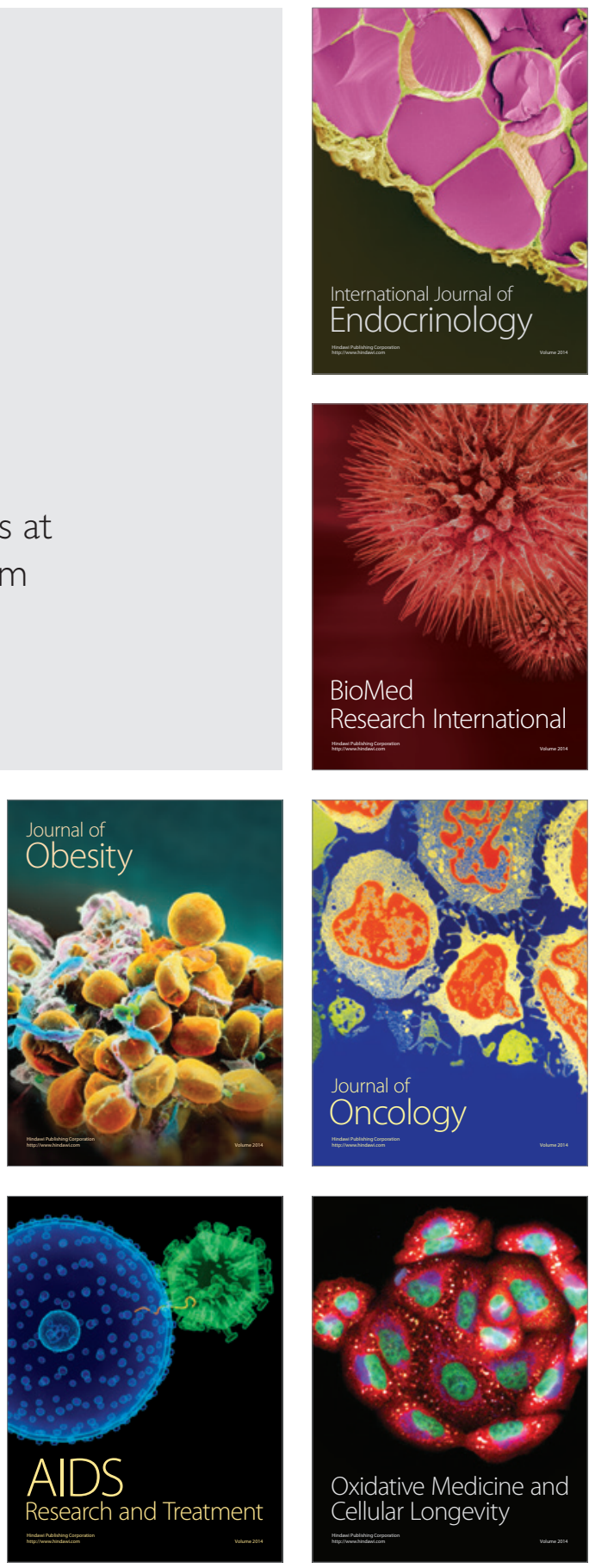